anti-MDA5 antibodies were more frequent in IMM-ILD group $(\mathrm{p}=0.006)$. In our IMM-ILD group we compared the autoantibodies with the clinical and serological features and we found that patients with IMM- ILD and anti Ro-52 antibody had more frequency of heliotrope rash $(p=0.045)$ and those with IMM- ILD and anti-Jo1 antibody had a higher level of CK $(p<0.001)$.

Table 1. Demographic characteristics

\begin{tabular}{lc}
\hline Age, mean SD & $40.611+/-16.37$ \\
Women, $\mathbf{n}(\%)$ & $24(66.7 \%)$ \\
Diagnosis & \\
$\quad$ Dermatomiositis & $25(69.4 \%)$ \\
Polimiositis & $6(16.7 \%)$ \\
$\quad$ Síndrome antisintetasa & $5(13.9 \%)$ \\
Time of evolution * & $6.00(2.25-19.00)$ \\
Interstitial lung disease & $11(30.6 \%)$ \\
Serological & \\
CK & 305.00 \\
& $(50.75-1969.250)$ \\
LDH & $460.727+/-384.76$ \\
Risk ILD complicated with IIM & $16(44.4 \%)$ \\
High & $8(22.2 \%)$ \\
Moderate & $12(33.3 \%)$ \\
Low & \\
\hline
\end{tabular}

*months

Table 2. Clinical and serological comparation between IMM groups with and without ILD

\begin{tabular}{lccc}
\hline & IMM with ILD, $\mathbf{n = 1 1}$ & IMM without ILD, $\mathbf{n = 2 5}$ & $\boldsymbol{p}$ \\
\hline Proximal muscle weakness & $10(90.9 \%)$ & $22(88.0 \%)$ & NS \\
Heliotrope Rash & $7(63.6 \%)$ & $10(40.0 \%)$ & NS \\
Mechanic hands & $4(36.4 \%)$ & $4(16.0 \%)$ & NS \\
Anti-Mi-2 & $2(18.2 \%)$ & $8(32.0 \%)$ & NS \\
Anti-Tif1 $y$ & $0(0.0 \%)$ & $6(24.0 \%)$ & NS \\
Anti-MDA5 & $4(36.4 \%)$ & $0(0.0 \%)$ & .006 \\
Anti-NXP2 & $1(9.1 \%)$ & $0(0.0 \%)$ & NS \\
Anti-SAE1 & $0(0.0 \%)$ & $1(4.0 \%)$ & NS \\
Anti-Ku & $0(0.0 \%)$ & $2(8.0 \%)$ & NS \\
Anti-PM/Sc1100 & $0(0.0 \%)$ & $1(4.0 \%)$ & NS \\
Anti-PM/Scl75 & $2(18.2 \%)$ & $0(0.0 \%)$ & NS \\
Anti-Jo1 & $1(9.1 \%)$ & $0(0.0 \%)$ & NS \\
Anti-SRP & $0(0.0 \%)$ & $3(12.0 \%)$ & NS \\
Anti- PL12 & $0(0 \%)$ & $1(4.0 \%)$ & NS \\
Anti-EJ & $1(0.1 \%)$ & $1(4.0 \%)$ & NS \\
Anti- PL7 & $1(9.1 \%)$ & $4(16.0 \%)$ & NS \\
Anti-OJ & $0(0.0 \%)$ & $2(8.0 \%)$ & NS \\
Anti-Ro52 & $5(45.5 \%)$ & $8(32.0 \%)$ & NS \\
& & &
\end{tabular}

Conclusion: Anti-MDA5 antibodies were more frequent in our IMM-ILD group than in IMM without ILD group. Almost half of our patients were in a high risk group, which means they need an early immunosuppressive treatment. In our IMM-ILD patients we found an association between the presence of anti Ro-52 antibody with heliotrope rash and of anti-Jo1 antibody to a higher level of CK.

References:

[1] Saketkoo, L. A., et al. (2010). Interstitial lung disease in idiopathic inflammatory myopathy. Current Rheumatology Reviews, 6(2), 108-119.

[2] H. Yoshifuji, "Biomarkers and Autoantibodies of Interstitial Lung Disease with Idiopathic Inflammatory Myopathies," Clin. Med. Insights Circ. Respir. Pulm. Med., vol. 9s1, pp. 141-147, 2015.

Disclosure of Interests: None declared

DOI: 10.1136/annrheumdis-2020-eular.5098

\section{AB0608 CARDIAC VESSELS CALCIFICATION IN A COHORT OF SYSTEMIC SCLEROSIS PATIENTS: POSSIBLE ROLE IN VASCULOPATHY AND HEART ABNORMALITIES.}

S. Sciacca ${ }^{1}$, C. Rotondo ${ }^{1}$, A. Corrado ${ }^{1}$, L. Giardullo ${ }^{1}$, S. Stefania ${ }^{1}$, A. Altomare ${ }^{1}$, F. P. Cantatore ${ }^{1} .{ }^{1}$ University of Foggia, Rheumatology Unit, Foggia, Italy

Background: Cardiovascular disease is the leading cause of morbidity and mortality worldwide. Myocardial calcifications have been related with cardiovascular diseases (CVD) such as focal wall motion abnormalities and arrhythmias. The impact of vascular calcifications is under investigation in order to define the risk of cardiovascular events. The relationship between cardiac calcification and systemic sclerosis (SSc) has not been investigated.

Objectives: The aim of the study is to evaluate the frequency of different patterns of cardiac calcification in SSc patients, and to correlate them to other CVD risk factors.
Methods: We analyzed thoracic-CT scanners of 35 SSc patients (88\% female, aged 47,8 ys $\pm 12,9$, disease duration 12,8 ys \pm 9 ) to determine the location and extension of vascular and cardiac calcification. All recruited patients fulfilled the 2013 ACR-EULAR classification criteria for SSc. No one patients had renal failure, cardiomyopathy, myocarditis, history of cardiac surgery or radiotherapy. Results: We found myocardial vessels calcifications (MCv) in 37\% SSc patients, aortic wall calcifications (ACW)in $60 \%$ SSc patients, cardiac valve calcifications (VC) in $28 \%$ SSc patient and heart wall calcifications (HCW) in $20 \%$.

The SSc patients with almost one calcification had older age $(65 \pm 9,8$ ys vs $50 \pm 8,8$ ys; $p=0,0001)$ and higher values of circulating NTproBNP $(336,9 \pm 351,9$ vs $144,2 \pm 107,8 ; p=0,04)$ compared to those without

In particular, the SSc patients with MCv had and uric acid $(5,3 \pm 1,5$ vs $4,1 \pm 1,3$; $\mathrm{p}=0,05)$, higher rate of $\mathrm{PAH}(25 \%$ vs $0 \% ; \mathrm{p}=0,037)$, arrhythmia $(38,5 \%$ vs $9 \%$; $\mathrm{p}=0,036)$ and higher prevalence of CENP-B antibodies $(46 \%$ vs $4 \% ; p=0,01)$ compared to patients without MCv.

Patients with $\mathrm{HCw}$ had lower $\mathrm{C}$ reactive protein $(0,16 \pm 0,10$ vs $0,7 \pm 0,7 ; p=0,008)$ compared to those without HCw. No differences in the rate of heart and vascula complications of SSc were observed.

The SSc patients with ACw had higher frequency of arrhythmia (33\% vs $0 \%$ $p=0,016)$ and longer disease duration $(15,5$ y $\pm 9,9$ vs $8,8 \pm 5,8 ; p=0,03)$.

The SSc patients with VC had higher rate of $\mathrm{PAH}(33 \% \mathrm{vs} 0 \% ; \mathrm{p}=0,003)$ and uric acid $(6 \pm 0,5 v s 3,8 \pm 1,2 p=0,0001)$.

Regression analysis excluded any association with gender, BMI, systemic arterial hypertension, steroid therapy, hypovitaminosis D or smoke habit. No cardiovascular event was recorded in one year of observation.

Conclusion: All patterns of calcifications may be related mostly with the older age. Myocardial vessels calcifications have been found in a high percentage of SSc patients and in particular in those with PAH and positive for anti CENP-B. Furthermore, myocardial vessels calcifications could be associated to the higher occurrence of arrhythmia. More studied are needed to assess the importance of vascular calcification as a part of the vascular involvement in SSc.

References:

[1] John W. Nance Jr. MD. Myocardial calcifications: Pathophysiology, etiologies, differential diagnoses, and imaging findings. Journal of Cardiovascular Computed Tomography 9 (2015) 58 e 67.

[2] Pagkopoulou E, Poutakidou M. Cardiovascular risk in systemic sclerosis: Micro- and Macro-vascular involvement. Indian J Rheumatol 2017;12, Suppl S1:211-7

[3] Plastiras SC, Toumanidis ST. Systemic sclerosis: the heart of the matter. Hellenic J Cardiol. 2012;53(4):287-300.

Disclosure of Interests: None declared

DOI: 10.1136/annrheumdis-2020-eular.5746

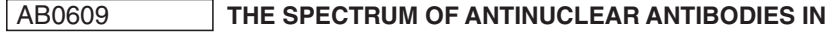 PATIENTS WITH SYSTEMIC SCLEROSIS POSITIVE FOR ANTI-U1RNP}

R. Shayakhmetova ${ }^{1}$, L. P. Ananyeva ${ }^{1}$, O. Koneva ${ }^{1}$, M. Starovoytova ${ }^{1}$,

O. Ovsyannikova', O. Desinova', L. Garzanova ${ }^{1} .{ }^{1}$ V.A. Nasonova Research

Institute of Rheumatology, Moscow, Russian Federation

Background: Patients with systemic sclerosis positive for anti-U1RNP have special clinical picture and disease progression. The autoimmune profile in this group is poorly understood.

Objectives: The purpose of our work was to study the level of major autoantibodies in patients with systemic sclerosis positive for anti-U1RNP.

Methods: The study included 80 patients $(71$ women and 9 men, mean age $44,5 \pm 14$ years) positive for antibodies to RNP and meeting the criteria of the systemic sclerosis (ACR/EULAR 2013). Patients were examined for autoantibodies: RF, ACCP, ACA, anti-Scl70, anti-RNAP-III, anti-Ro, anti-La, anti-dsDNA, anti-Sm, ACL, anti-Jo1. 44 patients were examined in dynamics in 24 months.

Results: In the study group the clinical picture was dominated by inflammatory musculoskeletal lesions (synovitis and myopathy), skin manifestations were poorly expressed. Interstitial lung disease was detected in $68 \%$ of cases. Overlaps (34\%) with other rheumatic diseases (rheumatoid arthritis, systemic lupus erythematosus) and combination with Sjogren's syndrome (32.5\%) were frequently noted. Other antibodies were often detected: commonly - RF (31\%), anti-Ro (38\%), anti-dsDNA (42\%), rarely - anti-Sm (11\%), ACCP (8\%), anti-La (8\%), ACA (6\%), anti-Scl70 (6\%), AKL (2\%). Anti-Jo1 and anti-RNAP-III were not detected at all. In patients with systemic sclerosis highly-positive for antiU1RNP (more than 2 upper normal limits) RF, anti-Ro, anti-dsDNA were significantly more common in comparison with low-positive $(\mathrm{p}=0.00)$. In dynamics $80 \%$ of patients maintained anti-U1RNP, while other autoantibodies were detected with the same frequency. In patients with initially low titer of anti-U1RNP, their disappearance was noted. 
Conclusion: Patients with systemic sclerosis positive for anti-U1RNP differ in the predominance of inflammatory musculoskeletal manifestations and frequent combination with Sjogren's syndrome and overlaps. Highly positivity for anti-U1RNP is accompanied by a persistent increase in RF, anti-Ro, anti-dsDNA

Disclosure of Interests: None declared

DOI: 10.1136/annrheumdis-2020-eular.5678

\section{AB0610 SEASONAL VARIATION IN IDIOPATHIC INFLAMMATORY MYOPATHIES INCIDENCE AND PRESENTATION: A RETROSPECTIVE STUDY IN BEIJING AND HONG KONG}

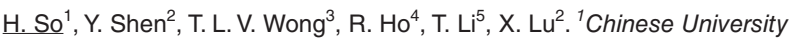
of Hong Kong, New Territories, Hong Kong (SAR); ${ }^{2}$ China Japan Friendship Hospital, Beijing, China; ${ }^{3}$ Kwong Wah Hospital, Kowloon, Hong Kong (SAR); ${ }^{4}$ Queen Elizabeth Hospital, Kowloon, Hong Kong (SAR); ${ }^{5}$ Queen Mary Hospital, Hong Kong, Hong Kong (SAR)

Background: Seasonal patterns of disease onset and severity in idiopathic inflammatory myopathies (IIMs) as a whole are conflicting [1-3]. In recent years, over 10 myositis-specific antibodies (MSAs) have been identified. They are able to divide patients into homogenous subgroups and inform on prognosis [4].

Objectives: The objective of the study was to investigate the seasonal variation of onset of IIMs characterised serologically.

Methods: This was a multi-centred retrospective observational study. Consecutive Chinese patients with IIMs admitted to the rheumatology wards of the participating major regional hospitals in Beijing and Hong Kong from July 2013 to June 2018 were recruited. The diagnosis of IIMs was based on the Bohan and Peter's criteria with definite or probable cases being included [5]. Patients with clinically amyopathic disease must have the typical Gottron's papules or heliotrope rash as determined by rheumatologists or dermatologists, and with no symptoms or signs of muscle involvement according to Sontheimer [6]. Patients with juvenile myositis, inclusion body myositis, cancer-associated myositis and myositis associated with other connective tissue disease were excluded. A commercial line blot immunoassay kit (EUROLINE) was used to detect the MSAs.

Results: All together 495 patients were studied. The mean age of the patients at disease onset was 48.1 years (S.D. 13.3). There was a female predominance (68.3\%). The subgroups of IIMs were: dermatomyositis (61.0\%), polymyositis $(21.8 \%)$, clinically amyopathic dermatomyositis $(12.9 \%)$, immune mediated necrotising myopathy $(3.8 \%)$ and nonspecific myositis $(0.4 \%)$. No particular seasonal pattern in disease onset was observed in IIM patients as a whole (Figure 1) or in any classical subgroups. However, significantly more patients with any one MSA had their disease started in the first half of the year $(p=0.007)$ as shown in Figure 2. Patients with either anti-synthetase or anti-MDA5 antibodies, which are associated with interstitial lung disease, had more frequent disease onset from November to February, which might coincide with the local flu season. It was also found that MSA positivity was associated with infection of the patient $(p=0.005)$. Further analyses showed that patients with MSAs which are typically associated with severe skin disease (MDA5, TIF1g, NXP2, SAE) had more hospitalisation from April to September where excessive sun exposure is expected. There were no major differences between the Beijing and Hong Kong subgroups.

Conclusion: Apparent seasonal patterns were noticed in our ethno-serologically defined IIM patients. Certain environmental factors, particularly infection or UV exposure, could be potential triggers. Our findings could shed light on the identification of etiologic factors and enhance our understanding of disease pathogenesis.

References:

[1] Manta P, Kalfakis N, Vassilopoulos D. Evidence for Seasonal Variation in Polymyositis. Neuroepidemiology 1989;8:262-265.

[2] Phillips BA, Zilko PJ, Garlepp MJ, et al. Seasonal occurrence of relapses in inflammatory myopathies: a preliminary study. J Neurol 2002;249:441-4.

[3] Lefe R, Burgess S, Miller F, et al. Distinct Seasonal Pattern in The Onset of Adult Idiopathic Inflammatory Myopathy in Patients with Auto Antibodies Anti-Jo-1 and Anti-Signal Recognition particle. Arthritis and Rheumatism 1991;34(11):1391-1396.

[4] Tansley SL, Betterridge ZE, McHugh NJ. The diagnostic utility of autoantibodies in adult and juvenile myostis. Curt Opin Rheumatol 2013;25(6):772-777.

[5] Bohan A, Peter JB. Polymyositis and dermatomyositis. N Engl J Med 1975;292:344-347.

[6] Sontheimer RD. Clinically myopathic dermatomyositis: what can we now tell our patients? Arch Dermatol 2010;146(1):76-80.
Figure 1.Disease onset of myositis patients.

50

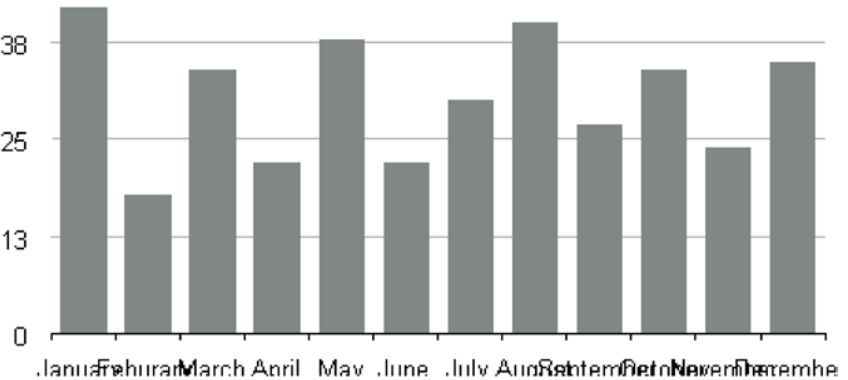

Figure 2. Disease onset of myositis patients with or without myositis specfic antibodies (MSA)

40

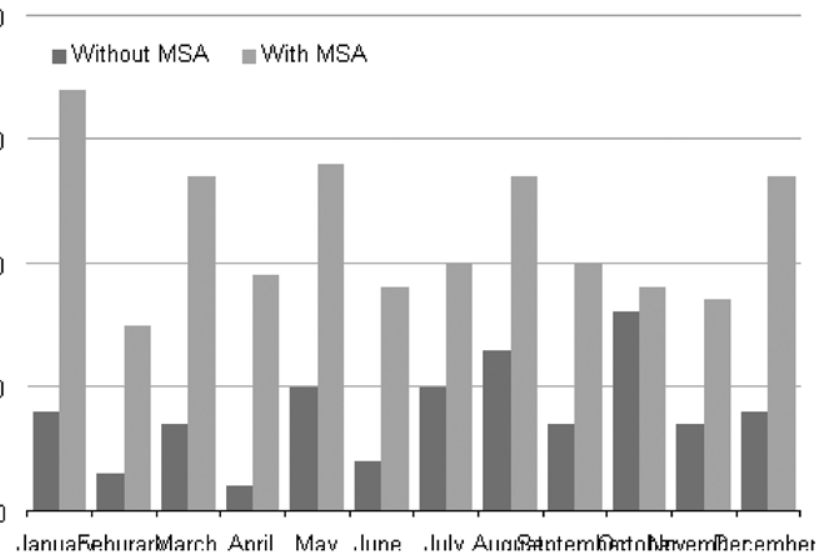

Disclosure of Interests: None declared

DOI: 10.1136/annrheumdis-2020-eular.5882

\begin{tabular}{|l|}
\hline AB0611 \\
STRAIN ANALYSIS OF THE RIGHT VENTRICLE USING \\
2D-SPECKLE TRACKING ECHOCARDIOGRAPHY IN A \\
COHORT OF PATIENTS WITH SYSTEMIC SCLEROSIS
\end{tabular}

A. Spinella ${ }^{1}$, P. Macripo, ${ }^{1}$, E. Cocchiara ${ }^{1}$, E. Galli ${ }^{1}$, F. Lumetti ${ }^{1}$, L. Magnani ${ }^{2}$, F. Coppi ${ }^{1}$, A. V. Mattioli ${ }^{1}$, R. Rossi ${ }^{1}$, G. Boriani ${ }^{1}$, C. Salvarani ${ }^{1}$, D. Giuggioli ${ }^{1}$.

${ }^{1}$ Policlinico of Modena University Hospital of Modena, Modena, Italy;

${ }^{2}$ Arcispedale Santa Maria Nuova, Reggio Emilia, Italy

Background: Systemic Sclerosis (SSc) is a rare and life-threatening connective tissue disease with multiple organ impairment. Cardio-pulmonary involvement is common: pulmonary fibrosis, pulmonary hypertension $(\mathrm{PH})$, and electrical disorders are the most serious complications and causes of increased mortality. Objectives: We evaluated features related with the onset and development of $\mathrm{PH}$ in a cohort of SSc patients. We further studied ecocardiographic abnormalities, by means of 2D-speckle tracking echocardiography (STE) with specific reference to the right ventricular strain measure (RV-strain).

Methods: We analyzed data from 50 SSc patients (pts) referred to our University-based Rheumatology Centre and SSc Unit from January 2007 to June 2019 (F/M 45/5; Ic/dcSSc 45/5; mean age 59.20 14.357 years; mean disease duration $12.08 \pm 8.75$ years). All pts underwent general and cardio-pulmonary assessment in our Cardio-Rheumatology Clinic. The following parameters were considered: blood exams, in particular inflammation indexes, uric acid test and serum autoantibodies; pulmonary function tests; high resolution scan of the lungs (HRCT); standard electrocardiogram (ECG) and RV-strain measured by 2D-STE. These examinations were performed according to clinical picture and current methodologies. We compared SSc subjects with (10/50) and without (40/50) PH diagnosis during follow-up regardless of treatments.

Results: SSc pts with $\mathrm{PH}$ didn't show significant alterations concerning RV-strain if compared with pts without PH ( $\mathrm{p}=0.707)$. Nevertheless, RV-strain value was modified in relation to TAPSE alterations in all pts but this data correlated with right ventricular dilatation only in $\mathrm{PH}$ subjects. Furthermore, interesting significant values about dilatation of right and left atria $(p=0.007, p=0.048)$, dilatation of inferior vena cava $(p=0.037)$ and right ventricle $(p=0.023)$ were observed. Left ventricular hypertrophy $(\mathrm{p}=0.012)$ as well as valvular insufficiencies (mitral and aortic) were more frequent in $\mathrm{PH}$ group too $(p=0.016)$. These pts showed higher incidence of 